\title{
ARTICLE Altered ErbB4 splicing and cortical parvalbumin interneuron dysfunction in schizophrenia and mood disorders
}

\author{
Daniel W. Chung ${ }^{1}$, Youjin Chung ${ }^{1,2}$, H. Holly Bazmi ${ }^{1}$ and David A. Lewis ${ }^{1}$
}

\begin{abstract}
Working memory requires the activity of parvalbumin (PV) interneurons in the dorsolateral prefrontal cortex (DLPFC). Impaired working memory and lower PV expression in the DLPFC are reported in schizophrenia and to a lesser degree in mood disorders. We previously proposed that activity-dependent PV expression is lower in schizophrenia due to a shift in the splicing of erb-b2 receptor tyrosine kinase 4 (ErbB4) transcripts from major to inactive minor variants that reduces excitatory drive to PV interneurons. Here, we tested the hypothesis that the degree of major-to-minor shift in ErbB4 splicing predicts the level of PV expression across schizophrenia and mood disorders. Levels of ErbB4 splice variants and PV mRNA were quantified by PCR in the DLPFC from 40 matched tetrads ( $N=160$ subjects) of schizophrenia, bipolar disorder (BD), major depressive disorder (MDD), and unaffected comparison subjects. Relative to unaffected comparison subjects, the magnitude of increases in minor variant levels and decreases in major variant levels was greatest in schizophrenia, intermediate in BD, and least in MDD. The same rank order was present for the magnitude of increases in the composite splicing score, which reflects the degree of major-to-minor shift across all ErbB4 splice loci, and for the magnitude of deficient PV expression. Finally, the composite splicing score negatively predicted PV expression across all subject groups. Together, these findings demonstrate a shared relationship between ErbB4 splicing and PV expression and suggest that scaling of the major-to-minor shift in ErbB4 splicing may influence the severity of deficient PV interneuron activity across diagnoses.
\end{abstract}

Neuropsychopharmacology (2018) 43:2478-2486; https://doi.org/10.1038/s41386-018-0169-7

\section{INTRODUCTION}

Impaired working memory is common in individuals with schizophrenia [1-3], bipolar disorder (BD) [4, 5] and major depressive disorder (MDD) $[6,7]$, and is an important determinant of long-term functional outcome and disability for individuals with these diagnoses $[5,8,9]$. Moreover, subjects with schizophrenia perform more poorly on measures of working memory than do subjects with mood disorders [10-12]. However, whether this difference in cognitive performance reflects scaling of a shared disease process across diagnoses is not well understood.

In schizophrenia, the neural substrate for working memory dysfunction is thought to involve reduced activity of parvalbumin (PV)-containing GABAergic interneurons in the dorsolateral prefrontal cortex (DLPFC) [13]. Levels of PV mRNA and protein, which are expressed in an activity-dependent manner and thus serve as markers of PV neuron activity [14, 15], have been consistently reported to be lower in the DLPFC of subjects with schizophrenia [16-24]. In contrast, PV levels have been found to be modestly lower or unaltered in the DLPFC of subjects with BD or MDD [16, 25-27]. Thus, the disease process that influences the severity of working memory dysfunction may be informed by investigating a putative pathogenic mechanism that could result in a range of deficits in PV interneuron activity across diagnoses.

The activity of PV interneurons is regulated, at least in part, by the neuregulin-1 (NRG-1)-erb-b2 receptor tyrosine kinase 4
(ErbB4) signaling pathway [28]. ErbB4, a member of the ErbB transmembrane receptor tyrosine kinase family [29], is highly enriched in the cell bodies and proximal dendrites of PV interneurons [30, 31]. The loss of ErbB4 signaling results in fewer excitatory synaptic inputs to, and reduced activity of, PV interneurons and consequently lower activity-dependent PV expression [32, 33].

The strength of ErbB4 signaling is regulated in part by alternative splicing of ErbB4 pre-mRNA. Splicing at the juxtamembrane (JM) locus generates the JM-a or JM-b variant based on the inclusion of exon 16 or $15 \mathrm{~b}$, respectively, whereas the inclusion or exclusion of exon 26 at the cytoplasmic (CYT) locus produces the CYT-1 or CYT-2 variant, respectively [34] (Fig. 1). Splice-specific in situ hybridization [35] and GPCR [18, 30] previously identified the JM-b/CYT-2 as the more common, major splice variant and the JM-a/CYT-1 as the less common, minor splice variant based on their relative expression levels in adult primate cortex. In response to NRG-1 stimulation, the major JM-b/CYT-2 variants display higher tyrosine kinase activity and result in a greater number of excitatory inputs to PV interneurons than do the minor JM-a/CYT-1 variants [36], suggesting that shifts in ErbB4 splicing from the major to minor variants would result in lower ErbB4 signaling, fewer excitatory inputs to and consequently lower activity of PV interneurons (Fig. 1). Consistent with this idea, a major-to-minor ErbB4 splicing shift has been reported in DLPFC gray matter from

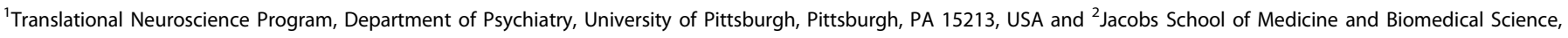
University at Buffalo, Buffalo, NY 14260, USA

Correspondence: David A. Lewis (lewisda@upmc.edu)

These authors contributed equally: Daniel W. Chung, Youjin Chung.
}

Received: 22 March 2018 Revised: 28 June 2018 Accepted: 25 July 2018

Published online: 2 August 2018 
A1

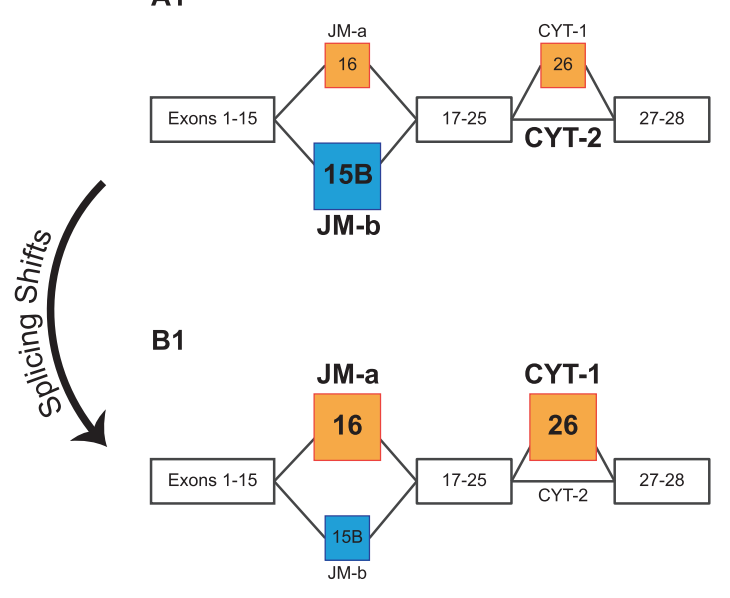

A2

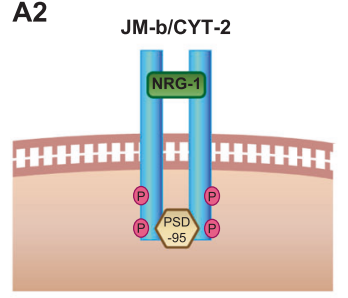

B2

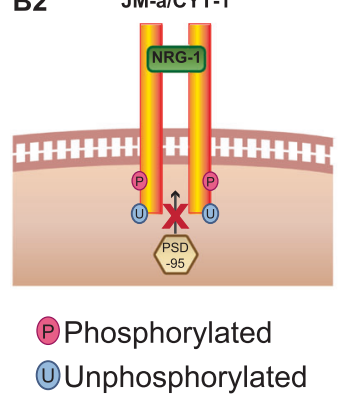

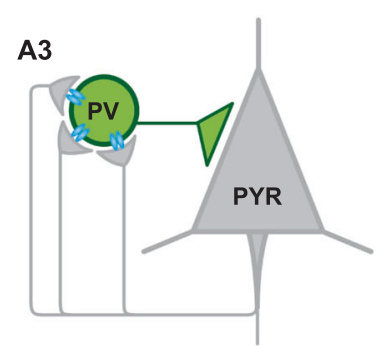

B3

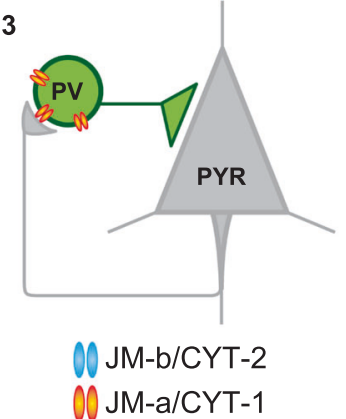

Fig. 1 Proposed role of ErbB4 splicing in regulating excitatory synaptic inputs to PV interneurons. a1 Alternative splicing at the juxtamembrane (JM) locus generates the minor JM-a or major JM-b variant based on the inclusion of exon 16 or $15 \mathrm{~b}$, respectively. Inclusion or exclusion of exon 26 at the cytoplasmic (CYT) locus produces the minor CYT-1 or major CYT-2 variant, respectively [34]. a2, a3 In response to neuregulin-1 (NRG-1), the tyrosine kinase domain of ErbB4 JM-b/CYT-2 variants auto-phosphorylate, which is required for the synaptic localization of PSD-95 and the formation of excitatory inputs to PV interneurons (Mei et al. 2014). b1 The alternative splicing of ErbB4 transcripts can be shifted from the major JM-b/CYT-2 to the minor JM-a/CYT-1 variants selectively in PV interneurons [18]. b2, b3 In response to NRG-1, the minor JM-a/CYT-1 variants display reduced auto-phosphorylation, resulting in fewer excitatory synapses on PV interneurons [36]. Thus, the shift in splicing from the JM-b/CYT-2 to JM-a/CYT-1 variants is proposed to result in lower ErbB4 tyrosine kinase activity, fewer excitatory inputs to and consequently lower activity of PV interneurons. Consistent with this idea, in schizophrenia a shift in ErbB4 splicing from major-to-minor variants is negatively correlated with the number of excitatory inputs to PV interneurons [17] and lower activitydependent expression of PV transcripts [18]

schizophrenia subjects [18, 37-39]. This shift was found in PV interneurons but not in calretinin interneurons [18], and was negatively associated with both the number of excitatory inputs to PV neurons [17] and lower PV mRNA levels [18] in the illness. Thus, the shift in ErbB4 splicing from major-to-minor variants might be a molecular determinant of deficient PV interneuron activity in schizophrenia.

In this study, we hypothesized that a scaling of the shift in ErbB4 splicing from major-to-minor variants predicts the magnitude of lower PV mRNA expression across subjects with schizophrenia, BD and MDD. To test this hypothesis, we first assessed the expression levels of ErbB4 splice variants in the DLPFC of 40 matched tetrads of subjects with schizophrenia, BD, or MDD and unaffected comparison subjects. Next, we compared the magnitude of the major-to-minor shift in ErbB4 splicing across subjects with schizophrenia, BD and MDD relative to unaffected comparison subjects. Finally, we assessed the relative deficits in PV mRNA expression and determined whether the shift in ErbB4 splicing predicts PV mRNA levels across the diagnostic groups.

\section{METHODS}

\section{Human Subjects}

Brain specimens $(N=160)$ were obtained during routine autopsies conducted at the Office of Allegheny County of Medical Examiner (Pittsburgh, PA) after consent was obtained from the next-of-kin. An independent team of clinicians made consensus DSM-IV diagnoses for each subject using the results of structured interviews with family members and review of medical records.
The same approach was used to confirm the absence of psychiatric diagnoses in the unaffected comparison subjects. All procedures were approved by the University of Pittsburgh Committee for Oversight of Research and Clinical Training Involving Decedents and Institutional Review Board for Biomedical Research.

The cohort used in this study consisted of 40 subject tetrads, with each tetrad composed of one subject with schizophrenia, BD or MDD and one unaffected comparison subject (Table S1). To reduce biological variance among groups, subjects in each tetrad were matched perfectly for sex and as closely as possible for age. Subject groups did not differ in mean age, postmortem interval (PMI), brain $\mathrm{pH}, \mathrm{RNA}$ integrity number (RIN), or tissue storage time at $-80^{\circ}$, but the proportion of black subjects was significantly greater in schizophrenia relative to the other subject groups (Table 1).

RNA processing

For each subject, fresh-frozen coronal tissue blocks containing right DLPFC area 9 were mounted in a cryostat. In zones where the cortex had been blocked perpendicular to the pial surface, the gray-white matter boundary was carefully scored with a scalpel blade. The scored gray matter area was photographed and the number of tissue sections $(40 \mu \mathrm{m})$ required to collect $\sim 30 \mathrm{~mm}^{3}$ of gray matter was calculated for each subject. The resulting number of sections were cut by cryostat, and total gray matter in the region of interest was isolated and collected into tubes containing Trizol reagent (Invitrogen, Carlsbad, CA) in a manner that ensured minimal white matter contamination and excellent RNA preservation. Total RNA was isolated from tissue homogenates via RNeasy 
Table 1 Summary characteristics of study subjects

\begin{tabular}{|c|c|c|c|c|c|c|c|c|c|c|c|}
\hline & \multicolumn{3}{|c|}{$\begin{array}{l}\text { Unaffected } \\
\text { comparison }\end{array}$} & & \multicolumn{2}{|l|}{ Schizophrenia } & \multicolumn{2}{|c|}{ Bipolar disorder } & \multicolumn{2}{|c|}{$\begin{array}{l}\text { Major depressive } \\
\text { disorder }\end{array}$} & \multirow[b]{2}{*}{$x^{2}$ test } \\
\hline & Number & & $\%$ & & Number & $\%$ & Number & $\%$ & Number & $\%$ & \\
\hline Sample size & 40 & & & & 40 & & 40 & & 40 & & \\
\hline Male & 22 & & 55 & & 22 & 55 & 22 & 55 & 22 & 55 & $X(3)=0, p=1.000$ \\
\hline Female & 18 & & 45 & & 18 & 45 & 18 & 45 & 18 & 45 & \\
\hline White & 37 & & 93 & & 28 & 70 & 39 & 98 & 38 & 95 & $X(3)=19.3, p<0.001$ \\
\hline \multirow[t]{2}{*}{ Black } & 3 & & 8 & & 12 & 30 & 1 & 3 & 2 & 5 & \\
\hline & \multicolumn{3}{|c|}{ Mean } & SD & Mean & $\mathrm{SD}$ & Mean & SD & Mean & SD & ANOVA \\
\hline \multicolumn{2}{|l|}{ Age (years) } & \multicolumn{2}{|l|}{46.8} & 12.8 & 47.4 & 12.2 & 45.8 & 12.4 & 45.5 & 11.7 & $F_{3,156}=0.20, p=0.895$ \\
\hline \multicolumn{2}{|l|}{ PMI (h) } & \multicolumn{2}{|l|}{19.5} & 5.6 & 19.9 & 7.4 & 20.9 & 7.0 & 19.2 & 5.8 & $F_{3,156}=0.47, p=0.703$ \\
\hline \multicolumn{2}{|l|}{ Brain pH } & \multicolumn{2}{|l|}{6.6} & 0.3 & 6.6 & 0.3 & 6.6 & 0.3 & 6.5 & 0.2 & $F_{3,156}=1.01, p=0.390$ \\
\hline \multicolumn{2}{|l|}{ RNA ratio } & \multicolumn{2}{|l|}{1.7} & 0.2 & 1.5 & 0.3 & 1.6 & 0.3 & 1.6 & 0.2 & $F_{3,156}=1.36, p=0.258$ \\
\hline \multicolumn{2}{|c|}{ RIN } & \multicolumn{2}{|l|}{8.0} & 0.6 & 8.0 & 0.6 & 7.9 & 0.6 & 8.0 & 0.5 & $F_{3,156}=0.35, p=0.787$ \\
\hline \multicolumn{2}{|c|}{ Storage time (months) } & 122.3 & & 55.5 & 131.6 & 64.0 & 125.0 & 57.0 & 128.0 & 48.5 & $F_{3,156}=0.20, p=0.898$ \\
\hline
\end{tabular}

kit (Qiagen, Valencia, CA) followed by complementary DNA (cDNA) synthesized from standardized dilutions of total RNA (10 ng/ $\mu$ l) for each sample. RNA integrity was measured using bioanalyzer (Agilent Bioanalyzer, Agilent Technologies, Santa Clara, CA).

Quantitative polymerase chain reaction ( $q P C R$ )

The qPCR was performed using Power SYBR Green Mastermix and ViiA 7 Real-Time qPCR system (Applied Biosystems, Foster City, CA). The primer sets for PV, four splicing variants of ErbB4 (JM-a, JM-b, CYT-1, CYT-2) and pan-ErbB4 used in this study have each been previously shown to yield a single band of the predicted size on a gel, an amplification efficiency $>98 \%$ in standard curve analyses and specific single products in dissociation curve analyses [18]. Beta actin, GAPDH and cyclophilin were used as reference genes as the expression levels of these genes in the DLPFC were previously reported to be stable in subjects with schizophrenia [40] and BD or MDD [27] relative to unaffected comparison subjects. In the present study, the geometric mean for the expression levels of beta actin, GAPDH and cyclophilin did not differ across schizophrenia, BD, MDD and unaffected comparison subjects $\left(F_{3,117}=1.9, p=0.130\right)$.

To control for experimental variance, cDNA samples from the matched subjects in each tetrad were amplified on the same 386 well plate. The difference in CT (dCT) for each target transcript was calculated by subtracting the geometric mean $\mathrm{CT}$ for the three reference genes from the $C T$ value of the target transcript. The expression level for each transcript was calculated as the expression ratio $\left(2^{-\mathrm{dCT}}\right)$. Consistent with our previous analysis of ErbB4 splice variant levels [18], we normalized the transcript levels of ErbB4 splice variants to the pan-ErbB4 levels within each subject to reduce the variability in ErbB4 splice variant levels attributable to any between-subject differences in total ErbB4 expression.

\section{Statistics}

Two analyses of covariance (ANCOVA) models were used to compare transcript levels across subject groups. The paired ANCOVA model included expression level as a dependent variable, subject tetrad as a blocking factor, and race, PMI, brain $\mathrm{pH}, \mathrm{RIN}$, and tissue storage time as covariates. This paired model accounts for the matching of tetrads for sex and age and for the parallel tissue processing of all subjects in a tetrad, but is not a true statistical paired design. Therefore, we also used an unpaired ANCOVA model which included expression level as a dependent variable and age, sex, race, $\mathrm{PMI}$, brain $\mathrm{pH}, \mathrm{RIN}$, and tissue storage time as covariates. Non-significant covariates were excluded in the final reported analyses. The paired and unpaired analyses from the ANCOVA produced comparable levels of statistical significance on all dependent variables except for the post hoc comparison on the $\mathrm{JM}-\mathrm{a}$ levels between BD and the unaffected comparison subjects. Thus, the main effect of diagnosis and the post hoc comparisons from the paired ANCOVA analyses are reported in the main text and the results from the unpaired ANCOVA analyses are provided in Table S2. The post hoc comparison of the unpaired ANCOVA analysis on the JM-a levels between BD and the unaffected comparison subjects is also reported in the main text.

To compare the magnitude of the major-to-minor shift in ErbB4 splicing across the diagnostic groups, the effect sizes of the dependent measures in each diagnostic group relative to the unaffected comparison subjects were calculated by Cohen's $d$ [41]. The $95 \%$ confidence intervals for the effect sizes were calculated as previously described [42].

The composite splicing score was computed using a previously described method [43] that provides equal weights to changes across all ErbB4 splicing loci. We first calculated the ratio of minor to major variant levels at each splice locus (i.e., JM-a:JM-b ratio and CYT-1:CYT-2 ratio). For each variant ratio, we calculated the $Z$ score $=((X-\mu) / \sigma)$, where $\mu$ is the mean of the group, $\sigma$ is the standard deviation of the group, and $X$ is the variant ratio for an individual subject. Then, we calculated the composite splicing score by summing the Z-scores of JM-a:JM-b ratio and CYT-1:CYT-2 ratio for each subject. The scores were normalized to the mean composite splicing score of the unaffected comparison subjects.

The potential influence of co-morbid factors that are frequently present in subjects with schizophrenia, BD or MDD (e.g., history of substance abuse, use of nicotine, antipsychotics, antidepressants, or benzodiazepines and/or sodium valproate at the time of death, or death by suicide), was assessed by ANCOVA models for each subject group with each factor as the main effect and age, sex, PMl, brain $\mathrm{pH}, \mathrm{RIN}$, and tissue storage time as covariates. A similar approach was used to assess an impact of psychosis in BD and MDD subjects or diagnosis of schizoaffective disorder in schizophrenia subjects. 


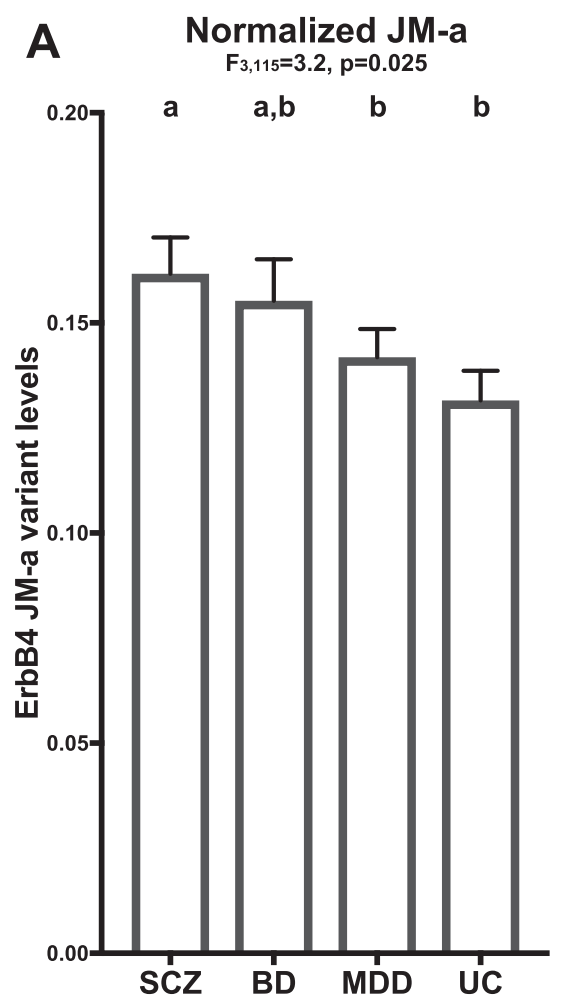

\section{B Normalized JM-b}
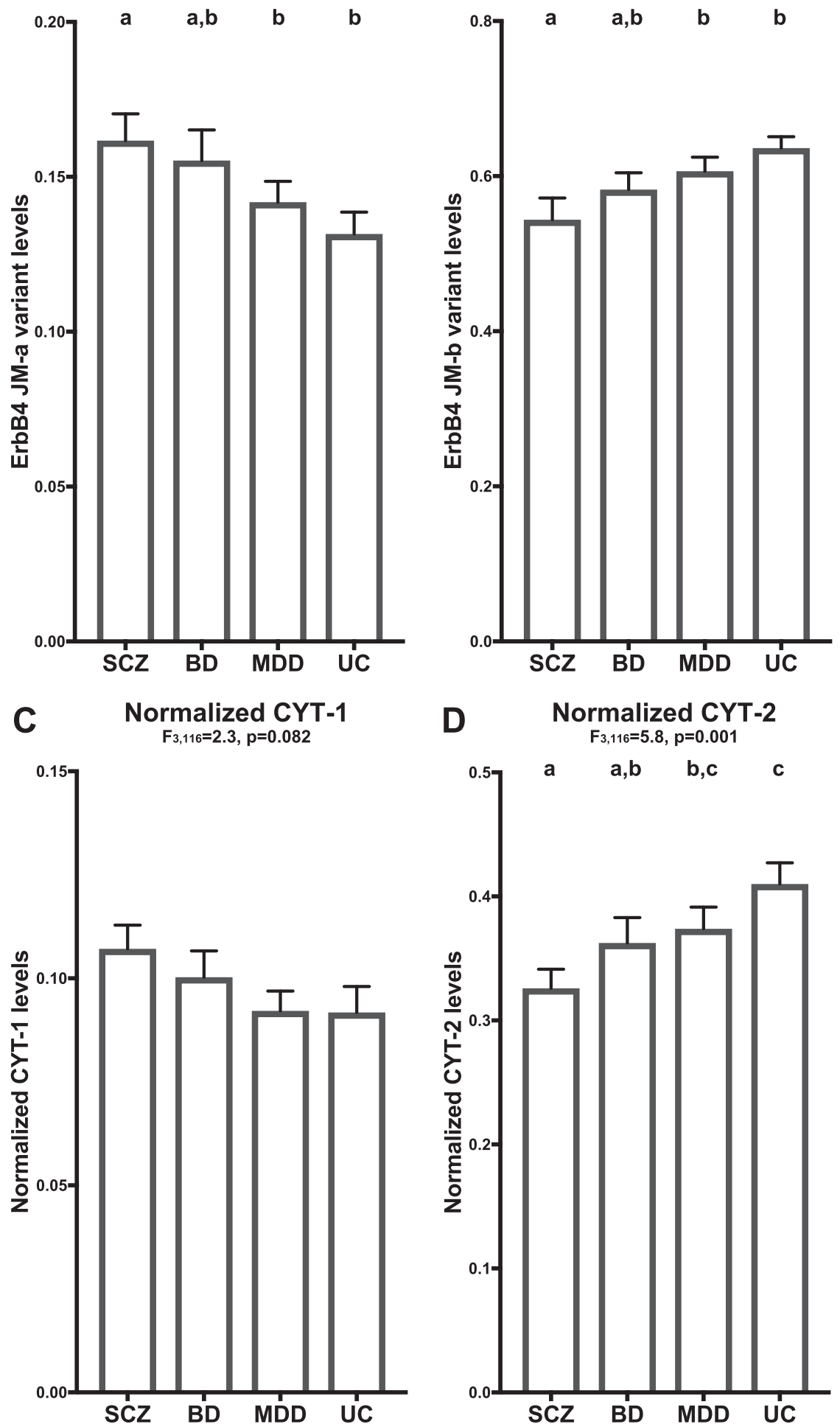

Fig. 2 Expression levels of ErbB4 splice variants in DLPFC area 9 of subjects with schizophrenia and mood disorders. Group mean (column) and standard error (bar) of JM-a (a), JM-b (b), CYT-1 (c), and CYT-2 (d) levels in subjects with schizophrenia (SCZ), bipolar disorder (BD), major depressive disorder (MDD), or unaffected comparison subjects (UC). Results of paired ANCOVA analyses are shown above each corresponding graph. Subject groups not sharing the same letter are significantly different $(p<0.05)$. The mean percent differences relative to unaffected comparison subjects in the variant levels were consistently greatest in schizophrenia (JM-a: $+28 \%$, JM-b: $-15 \%$, CYT-1: $+17 \%$, CYT-2: $-21 \%$ ), intermediate in BD (JM-a: +18\%, JM-b: $-8 \%$, CYT-1: +9\%, CYT-2: $-11 \%)$ and least in MDD subjects (JM-a: +8\%, JM-b: $-5 \%$, CYT-1: +1\%, CYT2: $-9 \%)$ 
Pearson's correlation analyses were performed to assess the relationships between the composite splicing scores and PV mRNA levels across subjects in each subject group as well as across all subjects.

Validation of the subject-matching strategy: impact of unaffected comparison subjects

We have previously reported higher JM-a and lower JM-b levels in total gray matter DLPFC of 62 schizophrenia subjects relative to their matched unaffected comparison subjects [18]; 36 of these schizophrenia subjects are included in the 40 tetrads used in the present study. However, 23 of those 36 schizophrenia subjects are matched here with unaffected comparison subjects that were not included in the original 62 pairs; this change was made to optimize the equivalence across all four subject groups of the variables shown in Table 1. Thus, to determine the potential impact of comparing schizophrenia subjects with different unaffected comparison subjects, we conducted a separate experiment to assess the levels of JM splice variants and PV mRNA in triads composed of these 23 schizophrenia subjects, their matched unaffected comparison subjects from the previous study and their matched unaffected comparison subjects from the current study. We found higher JM-a, lower JM-b and lower PV mRNA levels in the schizophrenia subjects relative to their matched unaffected comparison subjects from both studies (Figure $\mathrm{S} 1 \mathrm{~A}-\mathrm{C}$ ). These results demonstrate that dysregulated ErbB4 splicing and lower PV mRNA levels are consistently observed in schizophrenia, regardless of the subjects included in the unaffected comparison group.

\section{RESULTS}

Expression levels of ErbB4 splice variants in schizophrenia and mood disorders

In DLPFC area 9 of 40 matched tetrads of subjects with schizophrenia, BD, MDD and unaffected comparison subjects, mean normalized JM-a levels significantly differed across subject groups (Fig. 2a; $F_{3,115}=3.2, p=0.025$; See Figure S2 for plots showing values for individual subjects in each diagnostic group relative to their matched unaffected comparison subjects); relative to unaffected comparison subjects, the magnitude of the increase in JM-a levels was greatest in schizophrenia (effect size $=0.54$ ), intermediate in BD (effect size $=0.40$ ) and least in MDD subjects (effect size $=0.04$ ). Post hoc analyses revealed that JM-a levels in schizophrenia subjects were significantly increased relative to MDD $(p=0.019)$ and unaffected comparison subjects $(p=0.012)$ but not to BD subjects; JM-a levels in BD subjects were nearly significantly (Paired: $p=0.061$; Unpaired: $p=0.033$ ) increased relative to unaffected comparison subjects but not to MDD subjects; and JM-a levels in MDD subjects did not significantly differ from unaffected comparison subjects.

Mean normalized JM-b levels also significantly differed (Fig. 2b, Figure $S 2 ; F_{3,114}=3.1, p=0.029$ ) across subject groups; relative to unaffected comparison subjects, the magnitude of the decrease in $J M-b$ levels was greatest in schizophrenia (effect size $=0.63$ ), intermediate in BD (effect size $=0.37$ ) and least in MDD subjects (effect size $=0.10$ ). Post hoc analyses revealed that JM-b levels in schizophrenia were significantly decreased relative to MDD $(p=0.02)$ and unaffected comparison subjects $(p=0.007)$ but not to BD subjects; and JM-b levels in BD, MDD, and

B

B

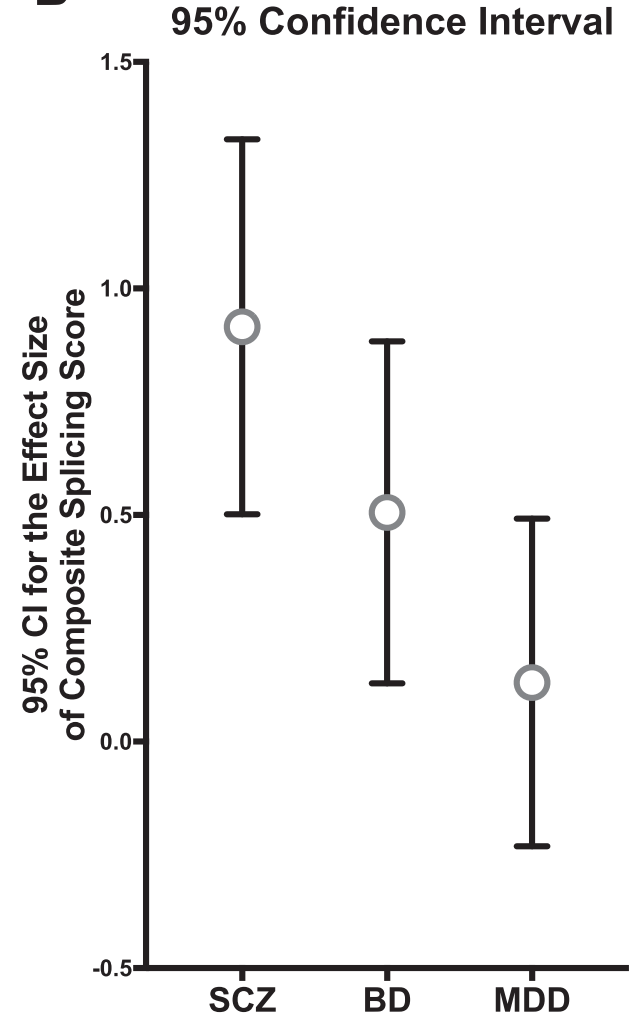

Fig. 3 Scaling of major-to-minor shift in ErbB4 splicing across schizophrenia and mood disorders. a Group mean (column) and standard error (bar) of the composite splicing score in subjects with schizophrenia (SCZ), bipolar disorder (BD), major depressive disorder (MDD), or unaffected comparison subjects (UC). Results of paired ANCOVA analyses are shown above the graph. Subject groups not sharing the same letter are significantly different $(p<0.05)$. b The effect size (circle) and its $95 \%$ confidence interval (bar) for the composite splicing score in subjects with schizophrenia (SCZ), bipolar disorder (BD), or major depressive disorder (MDD) relative to unaffected comparison subjects 
unaffected comparison subjects did not significantly differ from each other.

The main effect of diagnosis on mean normalized CYT-1 levels did not reach statistical significance (Fig. $2 \mathrm{c}$, Figure $\mathrm{S} 2 ; F_{3,116}=2.3$, $p=0.082$ ). However, relative to unaffected comparison subjects, the magnitude of the increase in CYT-1 levels was highest in schizophrenia (effect size $=0.35$ ), intermediate in BD (effect size $=0.19$ ) and least in MDD subjects (effect size $=0.16$ ).

Finally, mean normalized CYT-2 levels significantly differed (Fig. 2d, Figure S2; $F_{3,116}=5.8, p=0.001$ ) across subject groups; relative to unaffected comparison subjects, the magnitude of the decrease in CYT-2 levels was greatest in schizophrenia (effect size $=0.92$ ), intermediate in BD (effect size $=0.48$ ) and least in MDD subjects (effect size $=0.38$ ). Post hoc analyses showed that CYT-2 levels in schizophrenia subjects were significantly lower relative to $\operatorname{MDD}(p=0.017)$ and unaffected comparison subjects $(p<0.001)$, but not to BD subjects; CYT-2 levels in BD subjects were significantly decreased relative to unaffected comparison subjects $(p=0.032)$, but not to MDD subjects; and CYT-2 levels in MDD subjects did not significantly differ from unaffected comparison subjects.

In these analyses, there were significant effects of RIN on JM-a $\left(F_{1,115}=8.6, p=0.004\right)$, JM-b $\left(F_{1,114}=7.4, p=0.008\right)$, and CYT-2 $\left(F_{1,116}=5.1, p=0.025\right)$ levels, significant effects of brain $\mathrm{pH}$ on JMa $\left(F_{1,115}=24.8, p<0.001\right)$, JM-b $\left(F_{1,114}=8.7, p=0.004\right)$, and CYT-1 $\left(F_{1,116}=30.4, p<0.001\right)$ levels and a significant effect of storage time on JM-b levels $\left(F_{1,114}=8.6, p=0.004\right)$. Finally, neither the covariate effect of race across diagnoses nor the main effect of race within schizophrenia subjects was statistically significant (Table S3).

Effect of co-morbid factors on the ErbB4 splice variant levels Neither history of substance abuse; use of nicotine, antipsychotics, antidepressants, or benzodiazepines and/or sodium valproate at the time of death; nor death by suicide had a significant effect on expression levels of ErbB4 splice variants in subjects with schizophrenia, BD or MDD (Figures S3-5). Furthermore, neither a diagnosis of schizoaffective disorder in schizophrenia subjects (Figure S3) nor a history of psychosis in subjects with BD or MDD (Figures S4, S5) influenced ErbB4 splice variant levels.

Scaling of the major-to-minor shift in ErbB4 splicing across schizophrenia and mood disorders

Next, we assessed the magnitude of the shift in ErbB4 splicing at the JM or CYT locus across diagnostic groups. The ratio of minor to major variant levels (i.e., JM-a:JM-b ratio and CYT-1:CYT-2 ratio) was calculated to reflect the splicing shift at each locus. The ratios were log-transformed to normalize the distributions.

The mean JM-a:JM-b $\left(F_{3,115}=6.0, p=0.001\right)$ and CYT-1:CYT-2 $\left(F_{3,115}=6.1, p=0.001\right)$ ratios both significantly differed (Figure $\left.S 6\right)$ across subject groups; the magnitude of the increases in the JM-a: JM-b and CYT-1:CYT-2 ratios relative to unaffected comparison subjects was greatest in schizophrenia (effect size: JM ratio $=0.85$, $C Y T$ ratio $=0.88$ ), intermediate in $B D$ (effect size: $J M$ ratio $=0.51$, CYT ratio $=0.46$ ), and least in MDD subjects (effect size: $J M$ ratio $=$ 0.11 , CYT ratio $=0.13$ ). Post hoc analyses showed that the JM-a: JM-b and CYT-1:CYT-2 ratios in schizophrenia subjects were significantly higher relative to MDD (JM ratio: $p=0.001$; CYT ratio: $p=0.001$ ) and unaffected comparison subjects (JM ratio: $p<$ 0.001; CYT ratio: $p<0.001$ ), but not to BD subjects; the variant ratios in $\mathrm{BD}$ subjects were significantly higher relative to unaffected comparison subjects (JM ratio: $p=0.025$; CYT ratio: $p$ $=0.049$ ), but not to MDD subjects; and the variant ratios in MDD subjects did not significantly differ from unaffected comparison subjects.

We also computed the composite score of ErbB4 splicing by summing the normalized JM-a:JM-b and CYT-1:CYT-2 ratios (see Methods) in order to estimate the magnitude of the splicing shift
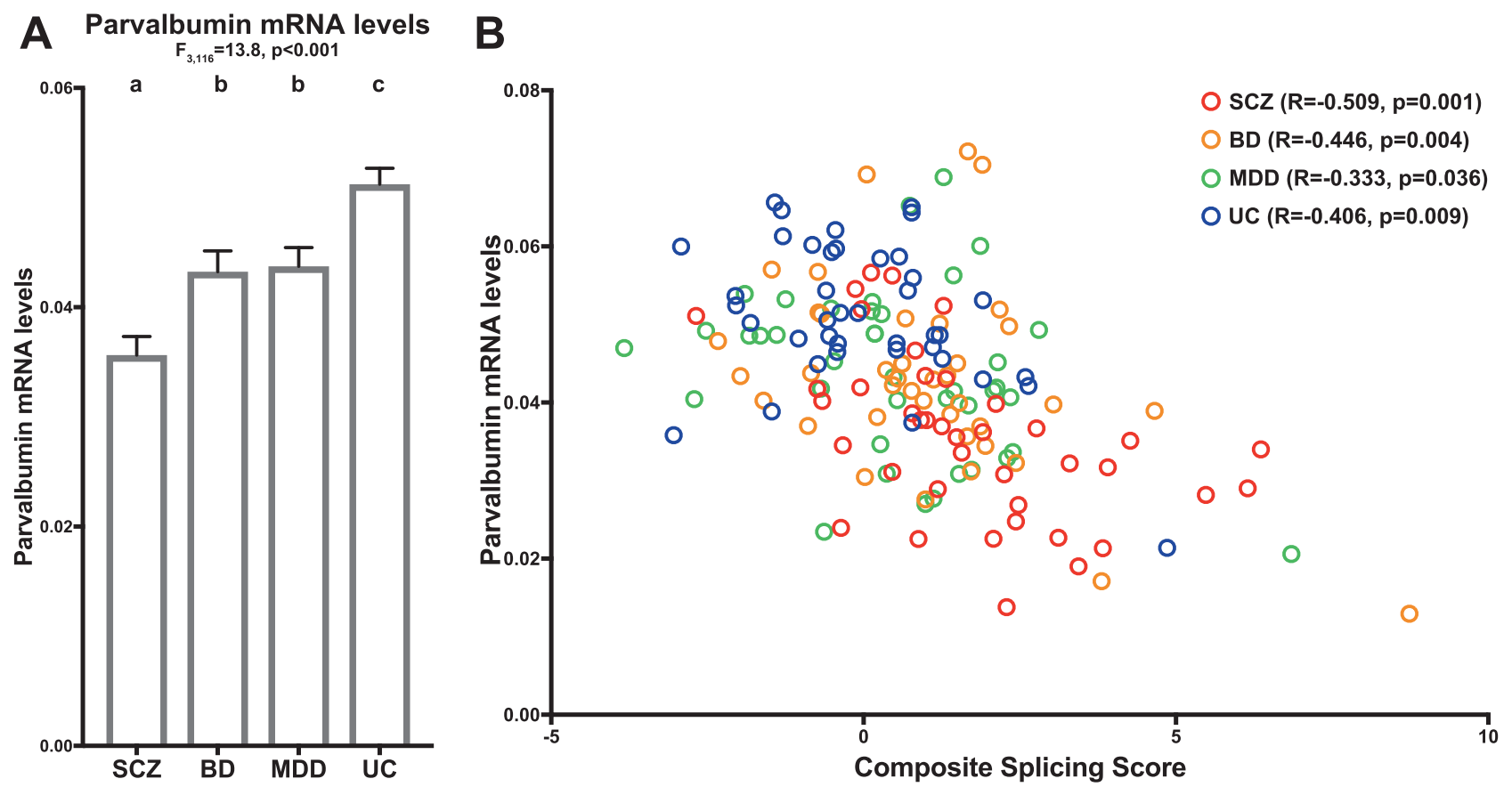

Fig. 4 Shared relationship between major-to-minor ErbB4 splicing shift and lower PV mRNA levels in schizophrenia and mood disorders. a Group mean (column) and standard error (bar) of parvalbumin mRNA levels in subjects with schizophrenia (SCZ), bipolar disorder (BD), major depressive disorder (MDD), or unaffected comparison subjects (UC). Results of paired ANCOVA analyses are shown above the graph. Subject groups not sharing the same letter are significantly different $(p<0.05)$. The mean percent decrease in parvalbumin levels was greatest in schizophrenia $(-30 \%)$, intermediate in BD $(-16 \%)$, and least in MDD subjects $(-15 \%)$. b Scatter plot of composite splicing scores relative to parvalbumin mRNA levels across all subjects $(N=160)$. The composite splicing score negatively predicted PV mRNA levels across all subjects as well as across subjects with schizophrenia (SCZ, red circles), bipolar disorder (BD, orange circles), major depressive disorder (MDD, green circles), or unaffected comparison subjects (UC, blue circles) 
from the JM-b/CYT-2 to the JM-a/CYT-1 variant. As expected, there was a significant effect of diagnosis on the composite splicing score (Fig. 3a; $F_{3,115}=6.8, p<0.001$ ); the magnitude of the increases in the composite splicing score relative to unaffected comparison subjects was greatest in schizophrenia (effect size $=$ 0.92 ), intermediate in $\mathrm{BD}$ (effect size $=0.51$ ) and least in MDD subjects (effect size $=0.13$ ). The $95 \%$ confidence interval for the effect sizes showed an overlap from MDD to BD and from BD to schizophrenia (Fig. 3b), reflecting a sequential increase in the magnitude of the major-to-minor variant shift from MDD to BD and from BD to schizophrenia. Post hoc analyses showed that the composite splicing score in schizophrenia subjects was significantly higher relative to $\operatorname{MDD}(p=0.001)$ and unaffected comparison subjects $(p=0.001)$, but not to BD subjects; the composite score in $\mathrm{BD}$ subjects was significantly higher relative to unaffected comparison subjects $(p=0.026)$, but not to MDD subjects; and the composite score in MDD subjects did not significantly differ from unaffected comparison subjects.

Shared relationship between ErbB4 splicing shift and PV mRNA levels across subject groups

Next, we assessed PV mRNA levels across subject groups. There was a significant main effect of diagnosis on mean PV mRNA levels (Fig. 4a, Figure S2; $F_{3,116}=13.8, p<0.001$ ). Post hoc analyses demonstrated that mean PV mRNA levels were significantly lower in schizophrenia $(p<0.001$; effect size $=1.39), \mathrm{BD} \quad(p=0.002$; effect size $=0.69$ ), and MDD ( $p=0.01$; effect size $=0.59$ ) subjects relative to unaffected comparison subjects. In addition, mean PV mRNA levels were significantly lower in schizophrenia relative to $\operatorname{BD}(p=0.002)$ and MDD $(p<0.001)$ subjects, but did not significantly differ between BD and MDD subjects.

In these analyses, none of the covariates had a significant effect on PV mRNA levels except for brain $\mathrm{pH}\left(F_{1,116}=19.2, p<0.001\right)$. There was a significant main effect of race on PV mRNA levels within schizophrenia subjects (Table S3); however, the mean PV mRNA level was $21 \%$ higher in black relative to white subjects in this group, suggesting that lower PV expression in schizophrenia relative to the other subject groups is not driven by a greater proportion of black subjects. There was no significant covariate effect of race across diagnoses on PV mRNA levels (Table S3).

Finally, we investigated if the shift in ErbB4 splicing from the JM-b/CYT-2 variant to the JM-a/CYT-1 variant predicted lower PV mRNA levels. Across all subjects, the composite splicing score was negatively correlated with PV mRNA levels (Fig. 4b; $R=-0.503, p$ $<0.001$ ). Within each group, the composite splicing score negatively predicted PV mRNA levels in subjects with schizophrenia $(R=-0.509, p=0.001), \mathrm{BD}(R=-0.446, p=0.004)$ or $\operatorname{MDD}(R=-0.333, p=0.036)$ and unaffected comparison subjects $(R=-0.406, p=0.009)$.

\section{DISCUSSION}

In this study, we tested the hypothesis that a scaling of the shift from major-to-minor variants in ErbB4 splicing predicts the magnitude of lower PV mRNA expression across subjects with schizophrenia, BD or MDD. We found that relative to unaffected comparison subjects, the decreases in major JM-b and CYT-2 variant levels, the increases in minor JM-a and CYT-1 levels, and their corresponding effect sizes were consistently least in MDD, intermediate in $\mathrm{BD}$ and greatest in schizophrenia. This rank order was also observed in the JM-a:JM-b and CYT-1:CYT-2 ratios, the composite splicing scores and their corresponding effect sizes. In concert, these results suggest that, relative to unaffected comparison subjects, the magnitude of shifts in ErbB4 splicing from major-to-minor variants increases from MDD to BD to schizophrenia subjects. In parallel to these changes, the deficits in PV mRNA levels and their corresponding effect sizes were greater in schizophrenia than in BD or MDD subjects. Finally, the composite splicing score negatively predicted PV mRNA levels across all subject groups. Together, these data demonstrate a shared relationship between the degree of major-to-minor shift in ErbB4 splicing and PV mRNA levels, suggesting that the scaling of ErbB4 splicing shifts influences the magnitude of deficits in PV expression across diagnoses.

Because PV expression is regulated in an activity-dependent manner [14, 15], our findings suggest that the scaling of major-tominor shifts in ErbB4 splicing could influence the severity of deficient PV interneuron activity across diagnoses. The ErbB4 major JM-b/CYT-2 variants display greater tyrosine kinase activity and produce a greater number of excitatory inputs to PV interneurons than do the minor JM-a/CYT-1 variants [36]. Thus, the major-to-minor shift in ErbB4 splicing would be expected to produce a corresponding decrease in the strength of ErbB4 signaling, resulting in fewer excitatory inputs to and lower activity of PV interneurons. Consistent with this idea, we have previously shown that the major-to-minor shift in ErbB4 splicing in PV neurons is associated with both fewer excitatory inputs to PV neurons and lower activity-dependent expression of PV transcripts in the DLPFC of schizophrenia subjects $[17,18]$. Consequently, the scaling of the shifts from major-to-minor variants in ErbB4 splicing could serve as a "molecular dial" that adjusts the strength of ErbB4 kinase activity to regulate the number of excitatory synaptic inputs to and the activity of PV interneurons across schizophrenia and mood disorders.

In addition to the ErbB4 splicing shift, other pathways are likely to influence the level of PV interneuron activity and thus PV expression in schizophrenia and mood disorders. For example, the neuronal activity-regulated pentraxin (NARP) regulates excitatory drive to PV interneurons [44]. NARP transcript levels are reduced to a similar degree in the DLPFC of subjects with schizophrenia, $\mathrm{BD}$ or MDD [45], suggesting that altered NARP signaling pathway could contribute to lower PV interneuron activity and PV expression across these disorders. Moreover, in NARP knock-out mice, the strength of ErbB4 signaling is unaltered [46], suggesting that these two pathways regulate PV interneuron activity independently. Thus, although altered NARP signaling may at least partly account for lower PV expression in schizophrenia and mood disorders, the differential reduction in PV expression across these diagnoses likely reflects the scaling of ErbB4 splicing shifts.

Reduced activity of PV interneurons is thought to contribute to the neural substrate for working memory impairment [13, 47]. Previous studies have shown that the individuals with schizophrenia perform significantly worse on measures of visual and spatial working memory than do individuals with mood disorders [10-12]. In parallel with this finding, our results suggest a more severe deficit in PV neuron activity in schizophrenia relative to mood disorders due to a greater shift in ErbB4 splicing. Thus, the difference in cognitive performance among individuals with schizophrenia and mood disorders may emerge, at least in part, from the scaling of major-to-minor variant shifts in ErbB4 splicing.

Our study suggests that shift in ErbB4 splicing is not due to experimental confounds or other factors commonly associated with schizophrenia or mood disorders. For example, although there was a greater proportion of black subjects in schizophrenia relative to the other subject groups, there was no significant effect of race on the expression levels of ErbB4 splice variants. Moreover, history of suicide, use of antipsychotic or antidepressant medications or substance abuse, which were reported in many subjects across diagnostic groups, did not have any effects on the ErbB4 splice variant levels. Finally, history of psychosis, a main diagnostic criterion of schizophrenia, was reported in $13 \mathrm{BD}$ subjects and 7 MDD subjects; however, there was no significant effect of psychosis on the variant levels in either subject group.

In conclusion, our study highlights a shared molecular basis for PFC dysfunction in schizophrenia and mood disorders. Moreover, 
it suggests that differences in the severity of deficient PV interneuron activity, and perhaps working memory impairment, across schizophrenia, BD and MDD may emerge, in part, from scaling of the major-to-minor variant shift in ErbB4 splicing. Thus, it is critical to investigate the upstream regulatory elements of ErbB4 splicing to further delineate the mechanism that adjusts the degree of shifts in alternative splicing across disorders.

\section{ACKNOWLEDGEMENTS}

We thank Kelly Rogers (University of Pittsburgh) and Mary Brady (University of Pittsburgh) for their technical assistance. Tissue samples from ten subjects used in this study were obtained from the NIH NeuroBioBank repository at the University of Pittsburgh. This work was supported by $\mathrm{NIH}$ grants MH043784 (to DAL) and $\mathrm{MH} 103204$ (to DAL). DAL currently receives investigator-initiated research support from Pfizer and serves as a consultant in the areas of target identification and validation and new compound development to Merck.

\section{ADDITIONAL INFORMATION}

Supplementary Information accompanies this paper at (https://doi.org/10.1038/ s41386-018-0169-7)

Competing interests: The authors declare no competing interests.

Publisher's note: Springer Nature remains neutral with regard to jurisdictional claims in published maps and institutional affiliations.

\section{REFERENCES}

1. Gold JM, Carpenter C, Randolph C, Goldberg TE, Weinberger DR. Auditory working memory and Wisconsin card sorting test performance in schizophrenia. Arch Gen Psychiatry. 1997;54:159-65.

2. Lee J, Park S. Working memory impairments in schizophrenia: a meta-analysis. J Abnorm Psychol. 2005;114:599-611.

3. Park S, Holzman PS. Schizophrenics show spatial working memory deficits. Arch Gen Psychiatry. 1992;49:975-82.

4. Bora E, Yucel M, Pantelis C. Cognitive endophenotypes of bipolar disorder: a meta-analysis of neuropsychological deficits in euthymic patients and their firstdegree relatives. J Affect Disord. 2009;113:1-20.

5. Martinez-Aran A, Vieta E, Reinares M, Colom F, Torrent C, Sanchez-Moreno J, et al. Cognitive function across manic or hypomanic, depressed, and euthymic states in bipolar disorder. Am J Psychiatry. 2004;161:262-70.

6. Kessler RC, Berglund P, Demler O, Jin R, Koretz D, Merikangas KR, et al. The epidemiology of major depressive disorder: results from the National Comorbidity Survey Replication (NCS-R). JAMA. 2003;289:3095-105.

7. Porter RJ, Gallagher $\mathrm{P}$, Thompson JM, Young AH. Neurocognitive impairment in drug-free patients with major depressive disorder. $\mathrm{Br} J$ Psychiatry. 2003;182:214-20.

8. Evans VC, Iverson GL, Yatham LN, Lam RW. The relationship between neurocognitive and psychosocial functioning in major depressive disorder: a systematic review. J Clin Psychiatry. 2014;75:1359-70.

9. Kahn RS, Keefe RS. Schizophrenia is a cognitive illness: time for a change in focus. JAMA Psychiatry. 2013;70:1107-12.

10. Bora $E$, Yucel $M$, Pantelis C. Cognitive impairment in schizophrenia and affective psychoses: implications for DSM-V criteria and beyond. Schizophr Bull. 2010;36:36-42.

11. Goldberg TE, Gold JM, Greenberg R, Griffin S, Schulz SC, Pickar D, et al. Contrasts between patients with affective disorders and patients with schizophrenia on a neuropsychological test battery. Am J Psychiatry. 1993;150:1355-62.

12. Reichenberg A, Harvey PD, Bowie CR, Mojtabai R, Rabinowitz J, Heaton RK, et al. Neuropsychological function and dysfunction in schizophrenia and psychotic affective disorders. Schizophr Bull. 2009;35:1022-9.

13. Lewis DA, Curley AA, Glausier JR, Volk DW. Cortical parvalbumin interneurons and cognitive dysfunction in schizophrenia. Trends Neurosci. 2012;35:57-67.

14. Patz S, Grabert J, Gorba T, Wirth MJ, Wahle P. Parvalbumin expression in visual cortical interneurons depends on neuronal activity and TrkB ligands during an Early period of postnatal development. Cereb Cortex. 2004;14:342-51.

15. Philpot BD, Lim JH, Brunjes PC. Activity-dependent regulation of calcium-binding proteins in the developing rat olfactory bulb. J Comp Neurol. 1997;387:12-26.

16. Beasley CL, Zhang ZJ, Patten I, Reynolds GP. Selective deficits in prefrontal cortical GABAergic neurons in schizophrenia defined by the presence of calciumbinding proteins. Biol Psychiatry. 2002;52:708-15.
17. Chung DW, Fish KN, Lewis DA. Pathological basis for deficient excitatory drive to cortical parvalbumin interneurons in schizophrenia. Am J Psychiatry. 2016;173:1131-9.

18. Chung DW, Volk DW, Arion D, Zhang Y, Sampson AR, Lewis DA. Dysregulated ErbB4 Splicing in Schizophrenia: Selective Effects on Parvalbumin Expression. Am J Psychiatry. 2016;173:60-8.

19. Enwright JF, Sanapala S, Foglio A, Berry R, Fish KN, Lewis DA. Reduced labeling of parvalbumin neurons and perineuronal nets in the dorsolateral prefrontal cortex of subjects with schizophrenia. Neuropsychopharmacology. 2016;41:2206-14.

20. Fung SJ, Webster MJ, Sivagnanasundaram S, Duncan C, Elashoff M, Weickert CS Expression of interneuron markers in the dorsolateral prefrontal cortex of the developing human and in schizophrenia. Am J Psychiatry. 2010;167:1479-88.

21. Glausier JR, Fish KN, Lewis DA. Altered parvalbumin basket cell inputs in the dorsolateral prefrontal cortex of schizophrenia subjects. Mol Psychiatry. 2014;19:30-6.

22. Hashimoto T, Volk DW, Eggan SM, Mirnics K, Pierri JN, Sun Z, et al. Gene expression deficits in a subclass of GABA neurons in the prefrontal cortex of subjects with schizophrenia. J Neurosci. 2003;23:6315-26.

23. Mellios N, Huang HS, Baker SP, Galdzicka M, Ginns E, Akbarian S. Molecular determinants of dysregulated GABAergic gene expression in the prefrontal cortex of subjects with schizophrenia. Biol Psychiatry. 2009;65:1006-14.

24. Volk DW, Matsubara T, Li S, Sengupta EJ, Georgiev D, Minabe Y, et al. Deficits in transcriptional regulators of cortical parvalbumin neurons in schizophrenia. Am J Psychiatry. 2012;169:1082-91.

25. Rajkowska G, O'Dwyer G, Teleki Z, Stockmeier CA, Miguel-Hidalgo JJ. GABAergic neurons immunoreactive for calcium binding proteins are reduced in the prefrontal cortex in major depression. Neuropsychopharmacology. 2007;32:471-82.

26. Sakai T, Oshima A, Nozaki Y, Ida I, Haga C, Akiyama H, et al. Changes in density of calcium-binding-protein-immunoreactive GABAergic neurons in prefrontal cortex in schizophrenia and bipolar disorder. Neuropathology. 2008;28:143-50.

27. Sibille E, Morris HM, Kota RS, Lewis DA. GABA-related transcripts in the dorsolateral prefrontal cortex in mood disorders. Int J Neuropsychopharmacol. 2011;14:721-34.

28. Mei L, Nave KA. Neuregulin-ERBB signaling in the nervous system and neuropsychiatric diseases. Neuron. 2014;83:27-49.

29. Bublil EM, Yarden Y. The EGF receptor family: spearheading a merger of signaling and therapeutics. Curr Opin Cell Biol. 2007;19:124-34.

30. Neddens J, Fish KN, Tricoire L, Vullhorst D, Shamir A, Chung W, et al. Conserved interneuron-specific ErbB4 expression in frontal cortex of rodents, monkeys, and humans: implications for schizophrenia. Biol Psychiatry. 2011;70:636-45.

31. Vullhorst D, Neddens J, Karavanova I, Tricoire L, Petralia RS, McBain CJ, et al. Selective expression of ErbB4 in interneurons, but not pyramidal cells, of the rodent hippocampus. J Neurosci. 2009;29:12255-64.

32. Del Pino I, Garcia-Frigola C, Dehorter N, Brotons-Mas JR, Alvarez-Salvado E, Martinez de Lagran $M$, et al. Erbb4 deletion from fast-spiking interneurons causes schizophrenia-like phenotypes. Neuron. 2013;79:1152-68.

33. Ting AK, Chen Y, Wen L, Yin DM, Shen C, Tao Y, et al. Neuregulin 1 promotes excitatory synapse development and function in GABAergic interneurons. J Neurosci. 2011;31:15-25.

34. Veikkolainen V, Vaparanta K, Halkilahti K, Iljin K, Sundvall M, Elenius K. Function of ERBB4 is determined by alternative splicing. Cell Cycle. 2011;10:2647-57.

35. Erben L, He MX, Laeremans A, Park E, Buonanno A. A novel ultrasensitive in situ hybridization approach to detect short sequences and splice variants with cellular resolution. Mol Neurobiol. 2017;55:6169-81.

36. Chung DW, Wills ZP, Fish KN, Lewis DA. Developmental pruning of excitatory synaptic inputs to parvalbumin interneurons in monkey prefrontal cortex. Proc Natl Acad Sci USA. 2017:114:E629-37.

37. Joshi D, Fullerton JM, Weickert CS. Elevated ErbB4 mRNA is related to interneuron deficit in prefrontal cortex in schizophrenia. J Psychiatr Res. 2014;53:125-32.

38. Law AJ, Kleinman JE, Weinberger DR, Weickert CS. Disease-associated intronic variants in the ErbB4 gene are related to altered ErbB4 splice-variant expression in the brain in schizophrenia. Hum Mol Genet. 2007;16:129-41.

39. Silberberg G, Darvasi A, Pinkas-Kramarski R, Navon R. The involvement of ErbB4 with schizophrenia: association and expression studies. Am J Med Genet B Neuropsychiatr Genet. 2006;141B:142-8.

40. Hashimoto T, Bazmi HH, Mirnics K, Wu Q, Sampson AR, Lewis DA. Conserved regional patterns of GABA-related transcript expression in the neocortex of subjects with schizophrenia. Am J Psychiatry. 2008;165:479-89.

41. Cohen J. Statistical power analysis for the behavioral sciences. 2nd ed. Hillsdale, NJ: Erlbaum Associates; 1988. p. 567.

42. Nakagawa S, Cuthill IC. Effect size, confidence interval and statistical significance: a practical guide for biologists. Biol Rev Camb Philos Soc. 2007;82:591-605.

43. Hoftman GD, Dienel SJ, Bazmi HH, Zhang Y, Chen K, Lewis DA. Altered gradients of glutamate and gamma-aminobutyric acid transcripts in the cortical visuospatial working memory network in schizophrenia. Biol Psychiatry. 2018;83:670-9. 
Altered ErbB4 splicing and cortical parvalbumin interneuron dysfunction... DW Chung et al.

44. Chang MC, Park JM, Pelkey KA, Grabenstatter HL, Xu D, Linden DJ, et al. Narp regulates homeostatic scaling of excitatory synapses on parvalbumin-expressing interneurons. Nat Neurosci. 2010;13:1090-7.

45. Kimoto S, Zaki MM, Bazmi HH, Lewis DA. Altered markers of cortical gammaaminobutyric acid neuronal activity in schizophrenia: role of the NARP gene. JAMA Psychiatry. 2015;72:747-56.
46. Pelkey KA, Barksdale E, Craig MT, Yuan X, Sukumaran M, Vargish GA, et al. Pentraxins coordinate excitatory synapse maturation and circuit integration of parvalbumin interneurons. Neuron. 2015;85:1257-72.

47. Gonzalez-Burgos G, Cho RY, Lewis DA. Alterations in cortical network oscillations and parvalbumin neurons in schizophrenia. Biol Psychiatry. 2015;77: 1031-40. 